\title{
Multilinguales
}

\section{Climbié de Bernard B. Dadié : Une rhétorique scripturale métachronique de l'afropolitanisme}

Climbié by Bernard B. Dadié: a metachronic scriptural rhetoric of afropolitanism

$$
\text { كليمبيي لبيرنار ب.داديي: بلاغة كتابية تغايرية للأفروبوليتانية }
$$

\section{Arsène Blé Kain}

\section{(2) OpenEdition}

\section{Journals}

\section{Édition électronique}

URL : http://journals.openedition.org/multilinguales/1073

DOI : $10.4000 /$ multilinguales. 1073

ISSN : 2335-1853

\section{Éditeur}

Université Abderrahmane Mira - Bejaia

\section{Référence électronique}

Arsène Blé Kain, «Climbié de Bernard B. Dadié : Une rhétorique scripturale métachronique de l'afropolitanisme », Multilinguales [En ligne], 9 | 2018, mis en ligne le 01 juin 2018, consulté le 21 décembre 2020. URL : http://journals.openedition.org/multilinguales/1073 ; DOI : https://doi.org/ 10.4000/multilinguales. 1073

Ce document a été généré automatiquement le 21 décembre 2020.

\section{(i) $8 \odot$}

Multilinguales est mise à disposition selon les termes de la Licence Creative Commons Attribution Pas d'Utilisation Commerciale - Pas de Modification 4.0 International 


\section{Climbié de Bernard B. Dadié : Une rhétorique scripturale métachronique de l'afropolitanisme}

Climbié by Bernard B. Dadié: a metachronic scriptural rhetoric of afropolitanism

$$
\text { كليمبيي لبيرنار ب.داديي: بلاغة كتابية تغايرية للأفروبوليتانية }
$$

\section{Arsène Blé Kain}

Dans Sortir de la grande nuit-Essai sur l'Afrique décolonisée (2010), Achille M'bembé révèle que l'itinérance, la mobilité et le déplacement sont des paradigmes majeurs hors desquels l'histoire culturelle du continent africain ne se comprendrait guère. Cette culture de la mobilité, visible à travers des migrations constitutives des diasporas et par des échanges commerciaux de tous genres, voire par l'esclavage et la colonisation, le conduit à concevoir une modernité contemporaine africaine fondée sur une sensibilité culturelle, historique et esthétique qu'il indique sous l'appellation néologique d' afropolitanisme, c'est-à-dire

La conscience de cette imbrication de l'ici et de l'ailleurs, la présence de l'ailleurs dans l'ici et vice-versa, cette relativisation des racines et des appartenances primaires et cette manière d'embrasser, en toute connaissance de cause, l'étrange, l'étranger et le lointain, cette capacité de reconnaître sa face dans le visage de l'étranger et de valoriser les traces du lointain dans le proche, de domestiquer l'infamilier. (M’bembé, $2010: 229$ )

Une telle appréhension de l'identité des Africains reposant sur une interpénétration de cultures raciales diverses semble au principe d'un roman de l'époque coloniale africaine comme Climbié (Dadié, 1956). L'œuvre s'inscrit, à la faveur des itinérances multiples des personnages, surtout celles du personnage éponyme, dans un esprit cosmopolitique saisissable ici sous l'angle afropolitain et non plus suivant les pratiques colonialistes ou panafricanistes. La question de fond consiste à cerner les dispositifs discursifs et idéologiques qui permettent de faire ressortir de l'ouvrage de Bernard B. Dadié les traces d'une pratique prématurée de l'afropolitanisme dès lors que les 
personnages semblent partager l'expérience de cultures raciales plurielles. En d'autres termes, dans quelle mesure et suivant quelles modalités l'afropolitanisme se déploie-t-il dans Climbié?

3 S'inscrivant dans une perspective à la fois socio-pragmatique et postcoloniale, la présente étude analyse une des optiques identitaires selon laquelle le roman de Dadié pourrait être lu. Elle révèle ainsi de prime abord que «la circulation des mondes" perceptible dans cette œuvre littéraire ne doit pas être interprétée comme une tentative de reconfiguration de l'idéal panafricaniste pour ensuite dévoiler que le jeu de la mobilité qui s'y déroule est plutôt l'indice prémonitoire d'une praxis caractéristique de l'afropolitanisme.

\section{« La circulation des mondes » dans Climbié : une tentative manquée de reconfiguration de l'idéal panafricaniste}

4 Pour Achille M’bembé (2010:227-228), le phénomène de «la circulation des mondes » présente deux faces : la dispersion, qui fait de l'Afrique un lieu de départ vers d'autres régions du monde et dont la conséquence actuelle est la conversion de nombreuses personnes d'origines africaines en citoyens de divers pays du globe, et l'immersion qui concerne des populations venant d'autres continents, désormais enracinées en Afrique. Ces populations ont perdu tout contact avec leurs origines (européennes ou asiatiques) et sont devenues des "bâtards culturels". Et quand bien même elles prétendraient à une certaine supériorité au nom de la race et marqueraient leur différence, voire leur mépris à l'égard de tout ce qui est "africain » ou "indigène ", la plupart de ces « étrangers » s'expriment dans des langues locales, connaissent et pratiquent certaines coutumes du pays, même s'ils vivent toujours dans des communautés relativement fermées et endogamiques.

Dans Climbié, cette "circulation des mondes " à l'œuvre à travers les itinérances des personnages laisse voir une certaine solidarité africaine convergeant vers une mentalité de la blackness ${ }^{1}$ bien que le roman se présente, à terme, comme une phénoménographie infructueuse du panafricanisme.

\section{Les itinéraires des personnages : de la solidarité africaine à la blackness}

6 La circulation, le mouvement sont des caractéristiques majeures du roman de Bernard Dadié. Cette mobilité est perceptible aux nombreux déplacements des personnages, surtout ceux du personnage éponyme. L'histoire narrée évolue, de fait, à partir des voyages de Climbié. Ces déplacements intimement liés à son parcours scolaire s'effectuent à la fois en Côte d'Ivoire, son pays d'origine, et dans un espace extranational, le Sénégal.

7 Dès l'entame de l'œuvre, l'on découvre Climbié au campement, chez son oncle N'Dabian. Dans ce campement présenté, par la suite, sous le nom de Boudéa, il aide son oncle à diverses tâches et apprend, à ses côtés, son métier d'homme (p. 8). Sur recommandation de tante Bèniè, son épouse, l'oncle décide d'inscrire son protégé dans une école afin qu'il ne "subisse [pas] le sort que [lui] imposa [son] oncle qui [le] cachait au 
moment du recrutement scolaire» (15). Climbié quitte ainsi le campement pour GrandBassam où, chaque jour un peu plus, il oublie la vie champêtre de Boudéa. Ses seuls soucis se résument désormais à l'obtention d'une bonne note, d'une bonne place à l'école; cette ardeur au travail lui vaut d'accéder à l'école régionale de Grand-Bassam qu'il quitte par la suite pour la ville de Bingerville lorsqu'il est admis à entrer à l'école primaire supérieure (E.P.S.).

8 Les déplacements de Climbié en Côte d'Ivoire s'effectuent essentiellement dans la région de Grand-Bassam et de Bingerville, deux villes du Sud-Est. Sa mobilité demeure restreinte à un cadre spatial bien plus régional que national. La naissance de l'école coloniale en Côte d'Ivoire particulièrement sur les côtes et le site de Grand-Bassam, à l'époque, capitale du pays, justifie certainement cette trajectoire du personnage de Climbié sous-tendue par une quête effrénée du savoir. Ayant subi avec succès le concours d'entrée à l'École Normale William Ponty, il se voit obligé d'abandonner l'espace national pour migrer à Gorée au Sénégal (102). Dans le paquebot qui l'y conduit, il a pour voisin Dossou, un élève dahoméen qui connait bien le parcours et qui lui sert, à l'occasion, de guide. Les deux voisins s'appellent mutuellement « fofo » (107), c'est-à-dire frères.

Climbié sort ensuite de William Ponty pour entrer dans la vie active comme commis d'administration à Dakar (119). Il y découvre une certaine égalité entre Blancs et Noirs et en profite pour fréquenter des lieux de divertissement communs aux Africains et aux Européens (119). Il échange avec de jeunes administrateurs des colonies venus expressément en Afrique sous la houlette du Front Populaire Français pour servir la nouvelle politique coloniale (120). Il se rend, dans le cadre syndical, à Tiaroye (131) et à Saint-Louis (159), avec comme compagnon de voyage, pour la première destination, Dassi, un jeune Togolais. Il est pourtant contraint d'arrêter ses pérégrinations au Sénégal pour retourner en Côte d'Ivoire où il vient d'être réaffecté.

Les multiples contacts humains qui se créent à partir des nombreux déplacements de Climbié sont symptomatiques de ce qu'Achille M’Bembé qualifie de «circulation des mondes ». Ils permettent de découvrir, entre les Noirs, une certaine solidarité raciale inscriptible dans la blackness.

11 Dans Climbié, la solidarité raciale apparaît dans le mode de vie des Africains. Déjà dans son pays natal, Climbié réalise une certaine communion avec les Africains venus d'autres pays. À preuve, à Grand-Bassam où il fait ses premiers pas à l'école, autour de Climbié, porteur du "symbole", des élèves dahoméens mêlés à leurs camarades éburnéens chantent à l'unisson: "Tu parles agni, je te donne le symbole / Ah! Ah! je te donne le symbole. (...) / Tu parles baoulé, je te donne le symbole, / Ah! Ah! je te donne le symbole ». (19)

12 Cette solidarité raciale définissable par le terme de blackness se perçoit également à travers la présentation de vieux Sénégalais établis en Côte d'Ivoire depuis leur jeune âge et qui n'ont plus jamais regagné leur pays :

Sous les amandiers de Cayenne, des joueurs de boules et de cartes, près d'eux de vieux Sénégalais en grands boubous, dans leur chaise longue, la grosse canne entre les jambes, parlaient du temps où, tout jeunes, ils mirent les pieds en Côte d'Ivoire. Aujourd'hui, ils n'ont presque plus de dents, ne sont jamais retournés chez eux. (57)

13 La complicité qui semble régner entre les Africains n'occulte cependant pas le fait que ceux-ci n'entretiennent pas toujours et forcément entre eux des rapports amicaux. La mentalité de la blackness que l'on croirait découvrir dans leurs relations d'entraide et de 
convivialité n'aboutit, par conséquent, pas à l'esprit panafricaniste auquel l'on s'attendrait logiquement, Climbié demeurant, à ce titre, une phénoménographie infructueuse du panafricanisme.

\section{Climbié, une phénoménographie infructueuse du panafricanisme}

Apparu vers la fin du XIXe siècle aux Caraïbes pour désigner un mouvement de révolte des Africains confrontés à l'esclavage en Amérique et à la domination coloniale des Européens en Afrique, le panafricanisme se présente, dans sa première version, comme un mouvement d'émancipation des Africains et signifie, pour Blyden et Garvey ${ }^{3}$, un retour des Noirs vers la terre de leurs ancêtres.

Perçu donc d'abord comme un mouvement politique qui promeut et encourage la pratique de la solidarité entre les Africains où qu'ils soient dans le monde, le panafricanisme apparaît par la suite comme une vision sociale, culturelle et politique de leur émancipation en vue d'unifier ceux du continent et de la diaspora. Le panafricanisme se présente d'ailleurs finalement comme une pratique nationaliste à grande échelle. Pour M'bonda,

Être nationaliste, c'est en effet, montrer un certain attachement à sa nation, aussi bien sous forme de loyauté préférentielle que sous forme de dévouement pour son devenir. Le panafricanisme correspond à peu près à la même préoccupation: œuvrer pour la libération de la totalité de l'Afrique du joug de la domination étrangère, et aussi pour son unité et son développement social, économique et politique (12).

Il va ainsi au-delà de la simple conscience unitaire noire qui caractérise la blackness pour s'afficher, en définitive, comme une politique globalisante d'unification des Noirs et de développement de l'Afrique tout entière.

Cette entreprise qui consiste à se prendre en charge en tant qu'un seul et même peuple, avec des problèmes spécifiques et des solutions spécifiques à y apporter, se découvre, par exemple, dans le roman de Dadié, lors de la grève générale des travailleurs noirs. Le secrétaire général de l'Union des syndicats s'adresse aux militants en tant qu'Africains d'abord : «Camarades Africains, nous menons cette lutte depuis longtemps. » (153)

Ce n'est pas non plus un hasard si Climbié retrouve le Togolais Dassi dont il a fait la connaissance pendant le voyage à Tiaroye et qui travaille pourtant désormais comme gendarme dans la fonction publique ivoirienne (203). Dans cette même optique, Bernard Dadié, établissant un lien entre les Africains et les tam-tams, avance ces propos empreints d'un véritable sentiment panafricaniste :

Je viens de comprendre que tout chez l'Européen dans ce pays, est un réflexe d'autodéfense contre le climat d'abord, ensuite contre les hommes, les manœuvres, l'intellectuel, l'enfant qui part à l'école, et plus encore contre les tam-tams. Il se passera, certes, un certain nombre d'années avant que l'enfant devienne un concurrent sérieux, si toutefois on lui en donne l'occasion. Mais le tam-tam.

Cherchant notre cœur, notre esprit, notre âme, afin de réaliser une assimilation totale, il se dit "Comment puis-je dominer ce continent, ces hommes, lorsque le tam-tam, tous les soirs, leur tient le langage ancestral, les relie au passé ?" [...] Il est certain que les jeunes gens ne comprennent pas, tous, votre langage. Mais d'instinct, ils répondent : "Présent !" Vous faites partie de la communauté [...].

Tam-tams des funérailles et tam-tams des jours de fêtes ! Vous avez beau jouer le 14 juillet $^{4}$ et le 11 novembre ${ }^{5}$, vous avez beau répéter des refrains émaillés de mots français, vous demeurez spécifiquement africains. (207) 
Cette conscience panafricaniste justifie assurément le fait que l'Ivoirien Climbié et le Dahoméen Dossou s'appellent mutuellement « $f \circ f o$ » (107), c'est-à-dire frères.

En réalité, Climbié n'est pas un roman de défense et d'illustration de la culture noire ; il n'est pas non plus une œuvre de promotion de la solidarité raciale noire. Certains Noirs sont, de fait, plus proches des Européens que de leurs frères de race. Un des meilleurs amis de Climbié est M. Targe, un photographe européen avec qui il échange régulièrement et dont la lettre adressée à Climbié clôt le roman (208). Les conflits entre Blancs et Noirs résultent certes des rapports antagoniques raciaux dus à la situation coloniale, mais ils sont surtout le fait de frictions naturelles qui naissent de la cohabitation des hommes. La réflexion de $\mathrm{M}$. Targe répondant à Climbié qui accuse les Blancs de racisme est, à cet effet, instructive :

Nous avons des moments de crise. Cela arrive à n'importe quel homme. Ça nous arrive en Europe, comme cela vous arrive ici, à vous. Ce sont des moments de cafard parce que, parce que... C'est le mauvais temps de l'homme [...]. Mais ça passe. Ainsi, moi, j'ai quinze ans de colonie, ce que je ne tolérais pas en France, je ne le tolère pas ici non plus [...]. Le drame, c'est de dire : moi je ne suis pas Blanc, moi je ne suis pas Africain ; mais après tout, est-ce que nous ne sommes pas des hommes au même titre? $(160-161)$

21 Sur Le Marie Paul, le bateau qui le ramène en Côte d'Ivoire, Climbié fait également la mémorable expérience de la sympathie des Blancs vis-à-vis d'un Noir, là où des Noirs le déconsidèrent. Il est traité avec beaucoup d'égards par M. Jeline, un matelot européen (177), alors que le cambusier et le cuisinier noirs le méprisent royalement :

Au repas de midi, à Climbié, passager de deuxième classe ayant accepté de voyager en entrepont, le cambusier ne sert ni pain ni vin, et le cuisinier, sur trois plats, ne lui en donne qu'un en lui disant : "Vous ne travaillez pas, vous n'avez pas le droit de manger" (178).

Pire, lorsqu'un matelot blanc tente de le raisonner, celui-ci se lance dans de violentes invectives contre Climbié : "Qu'il parte en cabine et on lui f... la paix. Pour le moment, il est en entrepont... et de plus une bouche inutile » (178).

L'inimitié entre les personnages noirs apparaît au grand jour à travers la situation de privilégiés Dahoméens en Côte d'Ivoire qui se vantent des progrès de leur pays, par rapport à la Côte d'Ivoire « sauvage » (54): «Les Dahoméens occupaient les meilleures places dans les bureaux, remplissaient les autres emplois de cousins, dans le même temps que l'on licenciait des autochtones». (54); d'où les tensions qui surgissent entre les deux communautés.

24 Mieux encore, dans le roman de Dadié, des Européens prennent ouvertement le parti des Noirs. Ainsi en est-il de l'Européenne qui, à Conakry en Guinée, défend son mari, un sergent indigène arrêté par jalousie par d'autres Européens :

Messieurs! Cela peut vous paraître étrange, à vous autres, que nous épousions des Nègres. Pour nous, cela n'a rien d'insolite. Durant cette guerre, dans notre région, ils nous ont aidés à vivre à une époque où l'on se demandait à tout instant ce que serait demain. Nous n'avions nous autres ni boys, ni vivres à discrétion. Il vous est loisible de faire du racisme. En France, nous connaissons ce mot parce qu'il est dans le dictionnaire. Il n'est pas dans nos actes, dans nos faits, dans nos pensées. Il n'existe pas. Je vous demande donc de libérer mon mari. (181)

La communion entre Blancs et Noirs se manifeste tout aussi à des occasions diverses, voire banales : des Européens supportent des équipes de football locales (191); Noirs et Blancs tirent ensemble sur les lignes jetées par des matelots pour prendre des poissons 
(178-179) ; des Noirs se font enrôler pour faire la guerre aux côtés de leurs amis européens (125).

Climbié n'est donc pas un roman de l'éloge ou de la promotion du panafricanisme dès lors que les rapports qu'entretiennent les Blancs et les Noirs demeurent, à bien des égards, empreints de cordialité. Les Noirs semblent, au demeurant, participer de deux cultures: l'une héritée des ancêtres (la culture de l'ici) et l'autre léguée par le colonisateur blanc (la culture de l'ailleurs). La réflexion faite par Climbié à Dassi pendant le déplacement en car pour Tiaroye corrobore cette identité multiple :

Et si j'affirmais à mon tour que nous devons lire beaucoup, peut-être plus qu'aucun autre peuple au monde, parce que nous sommes précisément à la croisée de deux civilisations ? (...). Nous, écartelés entre l'Européen qui a ses traditions et les vieux appuyés sur la coutume qui fait leur force, que devenons-nous? (133-134)

Le processus de créolisation dû au fait que les populations africaines ainsi décrites développent une culture transnationale inhérente aux pratiques d'itinérances, de migrations, de mouvements ressortit indubitablement de l'afropolitanisme, concept initié en 2005 par Achille M'Bembé. Climbié, roman de l'époque coloniale africaine qui présente les innombrables déplacements du personnage éponyme, apparaît, de ce fait, comme une pratique scripturale précoce de l'afropolitanisme.

\section{Le jeu de la mobilité dans Climbié : indice prémonitoire d'une praxis caractéristique de l'afropolitanisme}

La mobilité constitue, aujourd'hui, une dimension importante de la vie sociale, puisque c'est elle qui la structure. Elle est une caractéristique historique principale des peuples africains et implique des trajectoires qui font se retrouver le monde nègre partout, et donc les Africains partout. Achille M'bembé illustre bien cette situation de citoyens du monde quand il affirme «qu'il y a une partie de l'histoire africaine se trouvant ailleurs, hors d'Afrique : il y a également une histoire du reste du monde dont les Nègres sont, par la force des choses, les acteurs et dépositaires. » (228)

En conséquence,

Leur manière d'être au monde, leur façon d' "être monde ", d'habiter le monde, [...] s'est toujours effectué sous le signe sinon du métissage culturel, du moins de l'imbrication des mondes, dans une lente et parfois incohérente danse qu'ils n'ont guère eu le loisir de choisir librement, mais qu'ils sont parvenus, tant bien que mal, à domestiquer et à mettre à leur service. (2010 : 228-229)

En prenant ainsi à son compte ce paradigme majeur de l'afropolitanisme qu'est la mobilité, Climbié de Dadié n'est-il pas finalement une littérarisation avant-terme involontaire du concept d'Achille M’bembé ?

\section{La mobilité, un paradigme majeur de l'afropolitanisme}

31 Dans son acception générale, la mobilité renvoie à un déplacement, un transfert, une évolution d'une chose (matérielle et/ou non matérielle), d'une personne et des informations d'un point donné à un autre. Ce terme est, toutefois, loin de désigner aujourd'hui uniquement le déplacement réel dans l'espace en décrivant seulement les rapports que l'homme entretient avec cet espace; d'où la reconsidération des approches définitionnelles et méthodologiques de la notion par plusieurs théoriciens. 
Deleuze (1983) conçoit la mobilité selon une approche à partir de l'art du cinéma qui introduit de la vie dans l'image. Il s'agit d'un principe à la fois mental, psychique et physique. L'homme deleuzien est ainsi un homme réellement mobile, un homme cosmique qui perçoit l'univers en mouvement et en constante création, et dont l'identité devient alors universelle, cosmopolite.

Arjun Appadurai (2001) la détermine, en des termes génériques, comme l'ensemble des «flux globaux » dans lesquels l'on peut ranger les déplacements des populations et des biens culturels présentés en paysages (migrations des hommes, développement des médias de masse qui permettent d'avoir des informations du monde entier, circulation de toutes sortes d'idées liées aux imaginaires individuels et collectifs). Cette mobilité fait que les individus et les groupes sociaux ne sont plus liés à des localités intrinsèques (par exemple, des États-nations particuliers), mais qu'ils créent leurs locaux, et produisent, par conséquent, de nouveaux espaces identitaires déterritorialisés.

Zygmunt Bauman (2005), pour sa part, qualifie de modernité liquide le flux incessant de la mobilité actuelle de la vie. Dans la « société liquide », dit-il, l'unique référence qui est l'individu intégré par son acte de consommation n'a de place définie que par les choix individuels qu'il fait. Aussi son identité fluctue-t-elle au gré des exigences de flexibilité, de mobilité : elle n'est donc pas fixée ad vitam aeternam. rencontre, la négociation, la production de trajectoires que l'on peut suivre au fur et à mesure que les hommes et les biens se déplacent dans un espace physique ou imaginaire, la plupart du temps, transnational. Il occupe une place de choix dans le concept d'afropolitanisme de l'historien camerounais Achille M'bembé. La circulation, le déplacement, le mouvement sont, en effet, des paradigmes majeurs qui conduisent à la conscience afropolitaine, ce sentiment d'un ici imbriqué dans un ailleurs, aboutissement des déplacements physiques des personnes, mais aussi de ceux qu'elles effectuent au travers de leur imaginaire.

36 La mobilité (la circulation, les déplacements, le mouvement) demeure donc caractéristique de l'époque moderne africaine, et même de celle passée. À ce propos, Catherine Mazauric, épiloguant sur les déplacements du personnage de Climbié, déclare que ceux-ci « s'inscrivent sur la toile de fond d'une mobilité dans l'espace qui constitue antérieurement "une donnée fondamentale de l'Afrique sahélienne avant la colonisation et dans ses débuts" » (7). Reprenant d'ailleurs à son compte les propos de Jean-Loup Amselle, elle qualifie l'Afrique de l'Ouest où se meut Climbié d'« espace international » (25).

Climbié demeure ainsi dans cette logique de mobilité : les multiples déplacements du personnage principal à l'intérieur du pays natal, mais aussi en dehors de ce pays demeurent emblématiques de la «circulation des mondes» et dévoilent, à n'en point douter, l'expérience de mondes divers faite par les personnages du roman. Les personnages se fondent dans ces divers mondes qu'ils parcourent en partageant les cultures qui y sont véhiculées, tout en restant accrochés à la leur. L'affirmation de Jean Dérive qui stipule que «dans son principe, l'identité de [l'] espace francophone est fondée sur la force d'attraction centripète de son noyau de référence historique, à savoir la France envisagée comme métropole-étalon » (136) semble ainsi désuète.

38 La conscience afropolitaine n'est pas, en effet, une simple et banale attitude d'assimilation, d'acculturation; elle consiste plutôt, à travers l'expérience de la circulation, à intégrer le monde des autres au sien. En épousant le parcours 
mouvementé du personnage principal et en entremêlant de façon harmonieuse des peuples d'origines diverses, Climbié suit assurément un itinéraire identitaire afropolitain, bien que ce terme soit métachronique à l'époque coloniale où ce roman est paru.

\section{Climbié ou la textualisation prodromique de l'afropolitanisme}

39 Du parcours du personnage de Climbié, depuis son enfance jusqu'à son retour au pays natal, se dégage une trajectoire identitaire: celle d'un sentiment national qui, se muant, au fur et à mesure des déplacements des personnages, en une pensée apparemment panafricaine, finit par se présenter véritablement comme une conscience afropolitaine. Climbié répond effectivement aux caractéristiques essentielles de l' afropolitanisme, ancrées dans la mobilité. Les différents déplacements auxquels s'adonnent les personnages, surtout ceux qui s'effectuent dans un cadre extranational, permettent de forger chez ceux-ci une telle mentalité. Les personnages ne sont plus seulement nationaux de leur pays; ils ne sont ni africains, ni européens, puisqu'ils partagent deux cultures et se sentent des deux cultures.

C'est cette ambivalence de l'identité culturelle coloniale que développe Homi Bhabha dans sa théorie de l'hybridité. Pour lui, l'hybridité «ouvre, au sens métaphorique, un espace où se construit un objet colonial nouveau » qui n'est ni le colonisé ni le colonisateur, mais quelque chose d'intermédiaire qu'il qualifie de «troisième espace », c'est-à-dire un lieu où les puretés, polarités et essentialismes sont abolis (129-135).

Le déplacement extranational de Climbié qui impulse ceux des autres personnages implique un tel métissage. Il se déroule certes exclusivement entre la Côte d'Ivoire et le Sénégal, deux espaces africains apparemment identiques, mais, en réalité, les lieux représentés sont des ethnoscapes ${ }^{6}$ cosmopolitiques. Dakar, par exemple, se présente comme une ville créolisée où se rencontrent des Asiatiques, des Européens et des Africains de nationalités diverses. L'un des lieux de divertissement prisés qu'Européens et Africains ont en partage est, d'ailleurs, la propriété d'un Chinois (122).

Ce melting-pot qui dépasse le cadre purement mental conduit à un brassage de cultures et à un partage d'expériences culturelles diverses. Grâce à ces rencontres, les personnages du roman s'imprègnent des réalités culturelles des autres mondes. Leur identité en demeure affectée puisque ces expériences qui les enrichissent font qu'ils adoptent certaines pratiques qui s'y rapportent au final.

Les Sénégalais, par exemple, bénéficient du statut de citoyen français dont ils jouissent pleinement. En Côte d'Ivoire, pour l'entrée au cinéma, ils ont un traitement de faveur par rapport aux autres Noirs. Ils sont considérés au même titre que les Blancs : «Les Européens, les Syriens et les Sénégalais entraient par une autre porte, sans bousculade aucune. Ils restaient là, à regarder cette foule hurlante qui se battait pour voir le "magicien blanc" " (39). Ils se complaisent dans cette situation qu'ils ne dénoncent pas outre-mesure, se sentant parfaitement français quelque part. Cette conscience d'appartenir aussi bien au Sénégal qu'à la France permet de comprendre leur empressement à se faire enrôler pour combattre pour la France envahie lors de la grande Guerre: "Les bureaux de recrutement pendant des mois furent assiégés par des gens enthousiastes pressés d'aller se battre.» (125)

Les personnages de Climbié participent ainsi de la conscience afropolitaine, car, au détour de leurs déplacements, ils développent une culture transnationale. Cette culture nouvelle transcontinentale relève d'un véritable mélange de cultures diverses 
interdépendantes dans lesquelles se reconnaissent dorénavant les personnages et à partir desquelles s'ordonnance désormais leur vie. La distinction entre eux et les autres est donc brouillée.

L'interrogation que formule Climbié dans l'échange qu'il a avec Dassi, son ami togolais, est révélatrice de cette situation : "Nous, écartelés entre l'Européen qui a ses traditions et les vieux appuyés sur la coutume qui fait leur force, que devenons-nous?» (134). La réponse se trouve assurément dans la missive adressée à Climbié par M. Targe, en fin de roman :

Aujourd'hui, il faut, et nous le voulons, dans cette compénétration des races et des peuples, donner une place prépondérante à la connaissance, à la compréhension et à l'amour...

Je dis que nous devons aller de l'avant, tracer hardiment les routes que suivront les nouvelles caravanes, les caravanes de la fraternité dans un monde pacifié. (208)

Cette compénétration des races et des peuples dont parle si bien M. Targe fait participer, à leur insu certainement, les Occidentaux à la culture africaine qui semble les happer :

Climbié comprit qu'une nouvelle classe d'Européens montait, la classe des colons, mi-européens mi-africains, avec un langage spécial qui ira en s'affirmant. Ces hommes-là, en Afrique penseront toujours à leur village. Et revenus dans leur pays, ils seront pressés de retourner en Afrique, parce que chez eux, ils ont des attitudes qui souvent blessent l'entourage. Ils ont beau se rassembler dans les hôtels, dans leurs quartiers, multiplier les constructions en béton armé afin de se maintenir, l'Afrique gagne sur eux, les griffe, les marque à tout jamais. Ils ont le cafard en Afrique. Ils ont la nostalgie en Europe. Ils ne sont plus les hommes d'un continent... mais un genre de trait d'union. (179)

Les Blancs, de même que les Noirs en qui « ce même phénomène se remarque chez les jeunes Africains évolués qui détonnent dans leur propre milieu » (180), demeurent ainsi entre deux cultures : celle de l'ici et celle de l'ailleurs.

La première nuit de Climbié, de retour dans son pays natal, reste, par exemple, troublée: "Il voudrait de plain-pied se mettre au diapason des êtres et des choses...Car il se souvient encore de cette phrase de Nalba: "À ton retour ne sois pas comme les autres qui ne veulent plus nous reconnaître" »(190-191). Il s'accommode mal de son pays, malgré d'énormes efforts d'adaptation ou de réadaptation. Les innombrables questions qui affluent dans sa tête, à son arrivée au port d'Abidjan, démontrent bien cet état d'être : "Pourquoi est-il descendu? ... Pourquoi a-t-il quitté Dakar où il s'était creusé un lit, avait pris des habitudes? Il était devenu étranger à son pays... Qui le connaît encore dans ce pays?» (188-189). La rencontre d'une cousine qui le reconnaît (190) et son engagement comme meneur dans la grève qualifiée de "grève des produits agricoles" en Côte d'Ivoire qui lui vaut d'être emprisonné (193-199) finissent pourtant par indiquer son attachement à ce pays.

49 Une telle reconfiguration culturelle permet de postuler que Climbié de Bernard B. Dadié est une véritable rhétorique scripturale afropolitaine, même si ce terme demeure chronologiquement postérieur à la période coloniale africaine sous laquelle cette œuvre est écrite.

50 Cette double postulation identitaire se résume dans la pensée d'Achille M'bembé qui confirme que "l'identité s'origine dans la multiplicité et la dispersion; que le renvoi à soi n'est possible que dans l'entre-deux, dans l'interstice entre la marque et la démarque, dans la coconstitution» $(2006: 118)$.

51 Aussi conclut-il que 
La colonisation n'apparaît plus comme une domination mécanique et unilatérale qui force l'assujetti au silence et à l'inaction. Au contraire, le colonisé est un individu vivant, parlant, conscient, agissant, dont l'identité est le résultat d'un triple mouvement d'effraction, de gommage et de réécriture de soi (118)

La réécriture de soi, attitude sociale nouvelle de se savoir et de se sentir à la fois d'ici et d'ailleurs, et la rhétorique qui l'accompagne justifient, d'ailleurs, l'« éloge de la créolité » que font Glissant et Chamoiseau ${ }^{7}$ quand ils affirment que :

Ce n'est pas parce qu'une communauté accueille des étrangers, consent à leurs différences, même à leurs opacités, qu'elle se dénature ou risque de périr. Elle s'augmente au contraire, et se complète ainsi. Elle donne de l'éclat à ce qu'elle est, à ce qu'elle a, comme à ce qu'elle devient, et elle offre cet éclat qui de s'offrir reçoit.

(20) Bernard B. Dadié. Cette œuvre présente, en effet, le personnage éponyme dans ses déplacements aussi bien dans le lieu d'origine, la Côte d'Ivoire, que dans l'espace extranational symbolisé par le Sénégal. À travers l'expérience de plusieurs mondes faite par les personnages, le roman paraît remettre en selle l'idéal panafricaniste qui a cours depuis le XIXe siècle. Le séjour de Climbié au Sénégal qui laisse découvrir une certaine solidarité africaine moulée dans la blackness, cette conscience globale de la quête d'un mieux-être des Noirs, présume logiquement de cette aspiration panafricaniste.

Les relations qu'entretiennent entre eux les personnages noirs ne sont pourtant pas toujours empreintes de cordialité puisque de vives tensions règnent entre certaines communautés noires et de graves mépris faits à des Noirs sont le fait d'autres Noirs. Climbié ne mérite pas, à ce titre, d'être considéré comme un roman militant pour une cause panafricaniste. Le jeu de la mobilité qui le caractérise et qui fait que les personnages, et surtout le personnage principal, se retrouvent à la croisée de cultures raciales diverses et éprouvent une sensibilité culturelle de l'ici et de l'ailleurs atteste bien que Climbié est une rhétorique scripturale métachronique de l'afropolitanisme dès lors que la pratique afropolitaine qui y est décrite se déroule à l'époque coloniale, bien avant la consécration du terme au XXIe siècle par Achille M’bembé. Climbié donne ainsi en synthèse et en perspective à toute l'humanité une proposition cosmopolitique de l'Afrique où le local s'articule harmonieusement avec l'universel.

\section{BIBLIOGRAPHIE}

AMSELLE, J.-L., «Ethnies et espaces : pour une anthropologie topologique », dans AMSELLE, J.-L., м’вокоцо, É. (dir(s).), Au cour de l'ethnie. Ethnie, tribalisme et État en Afrique, La Découverte/Poche, Paris, 1999, pp. 24-26.

APPADURAÏ, A., Après le colonialisme. Les conséquences culturelles de la globalisation, Payot, Paris, 2001.

BAUMAN, Z., La vie liquide, Librairie Arthème Fayard, Paris, 2005.

BHABHA H. K., Les lieux de la culture, Payot, Paris, 2007 [1994]

Multilinguales, 9 | 2018 
CANUT, C., MAZAURIC, C., La migration prise aux mots - Mise en récits et en images des migrations transafricaines, Cavalier bleu, Paris, 2014.

DADIÉ, B., Climbié, Seghers, Paris, 1956.

DELEUZE, G., L'image-mouvement, Cinéma I, Minuit, Paris, 1983.

DÉRIVE, J., « Stratégies identitaires du roman francophone ", in Annales de l'Université de Savoie, Université de Savoie, 1998, pp. 135-154.

FOUQUET, T., «Construire la blackness depuis l'Afrique, un renversement heuristique », in Politique

africaine, Karthala, Paris, $\mathrm{n}^{\circ}$ 136, 2014, pp. 5-19

GLISSANT, É., CHAMOISEAU, P., Quand les murs tombent - L'identité nationale hors-la-loi ? Galaade -

Institut du tout-monde, Paris, 1997.

M'BEMBÉ, A., Sortir de la grande nuit. Essai sur l'Afrique décolonisée, La Découverte, coll. « Cahiers

libres », Paris, 2010.

M'BEMBÉ, A., «Qu'est-ce que la pensée postcoloniale ? »(Entretien), in Esprit. Pour comprendre la pensée postcoloniale, décembre 2006, pp. 117-133.

M'BONDA Ernest-Marie, «Intellectuels africains, patriotisme et panafricanisme : à propos de la fuite des cerveaux », dans MANGU M'Bata André (dir.), Nationalisme, panafricanisme et reconstruction africaine, Codesria, Dakar, 2006, pp. 11-22.

\section{NOTES}

1. Notion complexe et ambivalente, la blackness pourrait se traduire comme l'identité ou la conscience noire dans son rapport à ce que certains critiques qualifient de whiteness ou « conscience blanche dominante ». Il s'agit d'un cosmopolitisme noir (Fouquet, 2014).

2. À l'école coloniale, le symbole était un objet encombrant et souvent malodorant que l'instituteur remettait en signe de punition à un élève surpris à parler une langue nationale en lieu et place de la langue du colonisateur.

3. Edward Wilmot Blyden (1832-1912) est considéré comme l'un des pères du panafricanisme quand Marcus Mosiah Garvey (1887-1940) en est une des figures principales des mouvements adressés aux masses.

4. Le 14 juillet est la date officielle de la fête nationale de la République française.

5. Le 11 novembre rappelle la signature en 1918 de l'armistice qui marque la fin des combats de la Première Guerre mondiale, la victoire des Alliés et la défaite totale de l'Allemagne.

6. Appaduraï nomme ainsi «les paysages d'identité de groupe» (2005: 91). «Les ethnoscapes sont en quelque sorte les paysages que se constituent des groupes mouvants eu égard à leurs propres origines et aux avatars qu'ils subissent " (Appaduraï, 2005 : 16). Il s'agit de paysage des individus (touristes, immigrants, réfugiés, exilés, travailleurs immigrés, et autres personnes et groupes se déplaçant) qui contribuent à la fabrication du monde changeant dans lequel nous vivons.

7. Glissant et Chamoiseau cherchent à développer le concept de mondialité en vue de traduire, sur le plan politique et poétique, une nouvelle conception du monde qui serait fondée sur l'ouverture des cultures, un état de mise en présence des cultures vécu dans le respect du Divers. 


\section{RÉSUMÉS}

Climbié de l'écrivain ivoirien Bernard B. Dadié apparaît, a priori, comme un roman de la refondation de l'idéal panafricaniste du fait que « la circulation des mondes » à l'œuvre dans le récit laisse découvrir une solidarité africaine qui converge progressivement vers une mentalité de la blackness. Les rapports d'amitié ou d'inimitié qui s'y tissent se soustrayant cependant des considérations raciales, et les Noirs participant même de deux cultures, l'une héritée des ancêtres noirs et l'autre léguée par le colonisateur blanc, cette œuvre demeure, à ce titre, une phénoménographie infructueuse du panafricanisme et se présente, en définitive, comme la textualisation avant-terme de l'afropolitanisme, cette conscience identitaire de l'imbrication de l' ici et de l'ailleurs.

Climbié by the Ivorian writer Bernard B. Dadié appears as a panafricanist ideal refoundation novel since "circulation des mondes" in the tale shows an African solidarity gradually converging to a mentality of blackness. The woven friendship and enmity report evade racial thoughtfulness. Black people have two cultures: the first, black ancestors inherited and the second left by white colonizers. As such, this work is an unsuccessful phenomenography of panafricanism and appears ultimately as preterm textualization of afropolitanism, this sense of identity nesting of the here and the elsewhere.

\section{INDEX}

Mots-clés : idéal panafricaniste, Circulation des mondes, Solidarité africaine, Blackness, Afropolitanisme

المثال الأفريقي, تضامن أفريقي, تنقل العوالم, سواد, أفروبوليتانيةفهرس الكلمات المفتاحية:

Keywords : panafricanist ideal, Circulation des mondes, African solidarity, Blackness, Afropolitanism

\section{AUTEUR}

\section{ARSĖNE BLÉ KAIN}

Université Alassane Ouattara 